\title{
Computationally efficient method to construct scar functions
}

\author{
F. Revuelta, ${ }^{1}$ E. G. Vergini, ${ }^{1,2}$ R. M. Benito, ${ }^{1}$ and F. Borondo ${ }^{3, *}$ \\ ${ }^{1}$ Grupo de Sistemas Complejos and Departamento de Física, Escuela Técnica Superior de Ingenieros Agrónomos, \\ Universidad Politécnica de Madrid, E-28040 Madrid, Spain \\ ${ }^{2}$ Departamento de Física, Comisión Nacional de Energía Atómica, Avenida del Libertador 8250, 1429 Buenos Aires, Argentina \\ ${ }^{3}$ Departamento de Química, and Instituto de Ciencias Matemáticas CSIC-UAM-UC3M-UCM, Universidad Autónoma de Madrid, \\ Cantoblanco, E-28049 Madrid, Spain
}

(Received 15 November 2011; revised manuscript received 25 January 2012; published 24 February 2012)

\begin{abstract}
The performance of a simple method [E. L. Sibert III, E. Vergini, R. M. Benito, and F. Borondo, New J. Phys. 10, 053016 (2008)] to efficiently compute scar functions along unstable periodic orbits with complicated trajectories in configuration space is discussed, using a classically chaotic two-dimensional quartic oscillator as an illustration.
\end{abstract}

DOI: 10.1103/PhysRevE.85.026214

PACS number(s): 05.45.Mt, 03.65.Sq

\section{INTRODUCTION}

The development of chaos theory that took place in the 1960s brought back to the forefront of physics several long-forgotten issues. One of them was the semiclassical quantization of chaotic systems [1] whose difficulty was first pointed out by Einstein [2,3], who noticed the lack of a supporting invariant classical structure for the wave functions.

It was Gutzwiller who made the first significant contribution to this problem by developing a semiclassical theory [4] able to quantize Hamiltonian systems with chaotic classical dynamics. His celebrated trace formula, solely based on periodic orbit (PO) information, opened the door for the study of the connection between these special trajectories and the eigenvalues of such systems. This topic was extensively studied in subsequent theoretical and experimental work [1].

POs may also have a dramatic influence on the corresponding eigenfunctions, since they were found to be the origin in some of them of an enhanced quantum density localization ("scars"), as first discussed by Heller [5]. The idea [6] behind this unexpected phenomenon, namely, the building up of probability due to constructive interference by recurrences caused by the dynamics along an unstable PO, has been ever since very fruitful [7]. Bogomolny later demonstrated that this extra density can be obtained in the $\hbar \rightarrow 0$ limit by averaging in configuration space groups of eigenfunctions in an energy window [8], and the corresponding phase space version was investigated by Berry [9]. Other interesting aspects of scarring, such as the role of recurrences taking place through homoclinic and heteroclinic quantized circuits $[10,11]$, the influence of bifurcations (in systems with mixed dynamics) [12], or the scarring of individual resonance eigenstates in open systems [13], have also been considered. Scars have also been experimentally observed [14,15], and their relevance in growing fields, such as nanotechnology [16], optical microcavitities [17], optical fibers [18], or graphene sheets, [19] has been described in the literature.

As a consequence, there is at present a great interest in the construction of wave functions with probability density highly localized along unstable POs of a classically chaotic system.

*f.borondo@uam.es
This allows the fundamental study of quantum localization phenomena, having at the same time potential technological applications. Different methods have been described in the literature to compute such functions [20-23], which present advantages and shortcomings. For example, that based on averaging groups of eigenstates using the true quantum dynamics of the system [20] is of very general validity and applicability, but it fails when the PO is either very unstable and/or has very long period (although still smaller than the Heisenberg's time). The reason for this failure is readily understood from Heller's view of scarring [24], who clearly showed the role of the competition existing between recurrences and the dispersing dynamics near the unstable PO [6]. Recently, this difficulty was overcome by decomposing the motion in the vicinity of the PO into one of periodic nature and another of hyperbolic character [21-23]. Even though these techniques put on equal footing all POs, they require a detailed knowledge of the stable and unstable manifolds in the vicinity of the POs. Explicit expressions were nevertheless obtained for the stadium billiard, taking advantage of the ray dynamics, and the cat map. This method, as described in Ref. [22], was later applied to a case with a smooth potential $[25,26]$.

In this paper, we study the performance of this method to systematically construct scar functions localized over long, complicated POs of classically chaotic systems. As an illustration, we apply it to a quartic two-dimensional oscillator, which is a benchmark in the field of quantum chaos [20,25-27]. This method can deal with any unstable PO, no matter how complicated it is, in an easy and straightforward manner.

The organization of the paper is as follows. In Sec. II, we briefly discuss the system used in our calculation. In Sec. III we describe the method that will be used to compute the scar functions. In Sec. IV some results are presented and discussed. We conclude the paper by presenting our conclusions and final remarks in Sec. V.

\section{SYSTEM}

The model that we have chosen to study is the dynamics of a unit mass particle moving in a quartic potential

$$
H=\frac{1}{2}\left(P_{x}^{2}+P_{y}^{2}\right)+\frac{1}{2} x^{2} y^{2}+\frac{\varepsilon}{4}\left(x^{4}+y^{4}\right),
$$




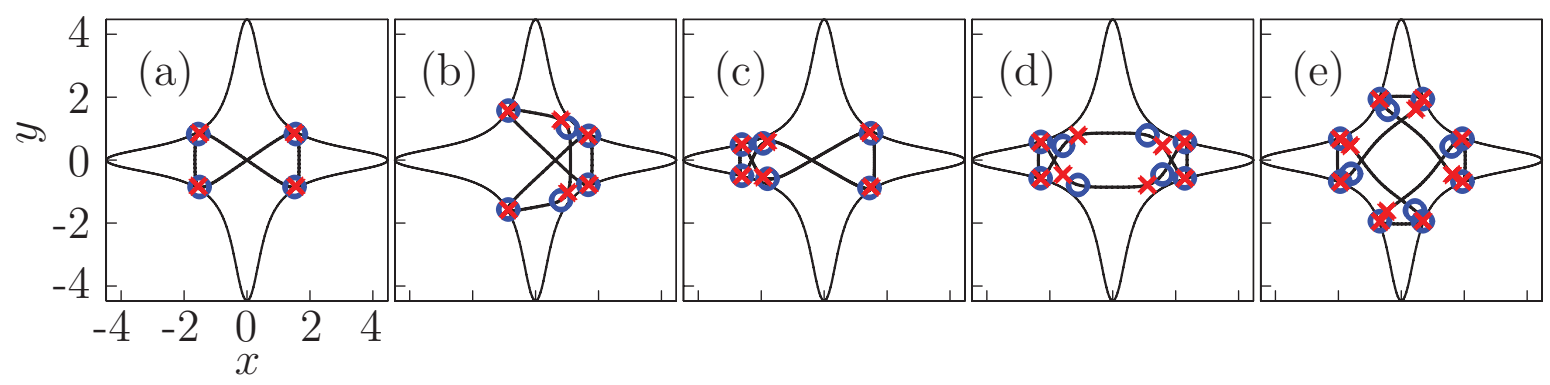

FIG. 1. (Color online) Some periodic orbits of the quartic oscillator for an energy $E=1$ that are used in the calculations of this paper. The blue circles and red crosses indicate the position of the self-conjugated points of the (clockwise) POs corresponding to the stable and unstable manifolds, respectively.

with $\varepsilon=0.01$. This system, whose dynamics are very chaotic [28], has been extensively studied [20,27] in connection with the issue of quantum chaos. The potential is homogeneous, which makes the corresponding classical dynamics mechanically similar. Indeed, any trajectory, $\left(x_{t}, y_{t}, P_{x, t}, P_{y, t}\right)$, at a given energy, $E$, can be scaled to another, $\left(x_{t^{\prime}}^{\prime}, y_{t^{\prime}}^{\prime}, P_{x, t^{\prime}}^{\prime}, P_{y, t^{\prime}}^{\prime}\right)$, at a different energy, $E^{\prime}$, by using the simple scaling relations

$$
\begin{aligned}
& x_{t^{\prime}}^{\prime}=\mathcal{E} x_{t}, \quad P_{x, t^{\prime}}^{\prime}=\mathcal{E}^{2} P_{x, t^{\prime}}, \quad t^{\prime}=\mathcal{E}^{-1} t, \\
& y_{t^{\prime}}^{\prime}=\mathcal{E} y_{t}, \quad P_{y, t^{\prime}}^{\prime}=\mathcal{E}^{2} P_{y, t^{\prime}}, \quad S_{t^{\prime}}^{\prime}=\mathcal{E}^{3} S_{t},
\end{aligned}
$$

with $\mathcal{E}=\left(E^{\prime} / E\right)^{1 / 4}$, and the dynamical action $S=$ $\int_{0}^{t} d \tau\left(P_{x, \tau} \dot{x}_{\tau}+P_{y, \tau} \dot{y}_{\tau}\right)$. This avoids the hassles associated with bifurcations or any other structural changes of the involved phase space.

We conclude this section by presenting in Fig. 1 some POs of Hamiltonian (1) that are used in the calculations presented in this paper. These POs have been selected since they constitute representative examples with different characteristics and complexity in the configuration space. As we progress toward the right, the complexity of the chosen PO in the configuration space increases. Actually, the last four [(b)-(e)] have been selected as describing rather complicated trajectories in the configuration space, making the application of the techniques to compute scar functions a priori difficult, if not impossible. Notice that in this spirit, we have not considered here POs which are too simple, such as for example those running along the axis or the diagonals, although our method works equally well in those cases.

\section{METHOD}

In this section we describe the method that will be used to systematically compute scar functions highly localized along any PO for a given degree of excitation along it.

The construction of the desired functions starts by defining tube functions with the probability density distributed along the PO as

$$
\psi_{n}^{\text {tube }}(x, y)=\int_{0}^{T} d t e^{i E_{n} t / \hbar} \phi(x, y, t),
$$

where $T$ is the period of the PO, and $E_{n}$ is the associated BohrSommerfeld (BS) quantized energy (see below). The function $\phi(x, y, t)$ is a suitable wave packet, whose probability density is forced to stay on the neighborhood of the PO, $\left(x_{t}, y_{t}, P_{x, t}, P_{y, t}\right)$.
For this purpose, we use a frozen Gaussian [29,30] centered on the trajectory,

$$
\begin{aligned}
\phi(x, y, t)= & \exp \left\{-\alpha\left[\left(x-x_{t}\right)^{2}+\left(y-y_{t}\right)^{2}\right]\right. \\
& \left.+\frac{i}{\hbar}\left[P_{x, t}\left(x-x_{t}\right)+P_{y, t}\left(y-y_{t}\right)\right]+i \gamma_{t}\right\},
\end{aligned}
$$

where $\alpha=1$ is adequate for our calculations. It should be noticed that when the guiding PO of this wave packet is not a librational or self-retracing trajectory, i.e., does not have turning points (TPs), the integrand in Eq. (3) should include two contributions, corresponding to the two opposite directions in which the PO can be run. The associated exponential factors are then complex conjugated, thus rendering an overall real expression for $\psi_{n}^{\text {tube }}$.

The phase accumulated during the propagation of $\phi(x, y, t)$ consists of two contributions: $\gamma_{t}=\left(S_{t} / \hbar\right)-(\pi / 2) \mu_{t}$, where the first term is proportional to the mechanical action, and the second one is a topological phase, that can be calculated by numerically computing the angle swept by the manifolds emanating from the "guiding" PO in the (phase space) plane transverse to it, being equal to $\mu_{t} \pi$. The value of $\mu_{\mathrm{T}} \equiv \mu$ after a full PO period is equal to the Maslov index [31] appearing in Gutzwiller's trace formula [32]. This quantity is simply given by the sum of the number of self-conjugated points (SCPs), generated by the rotation of either the stable or the unstable manifolds, plus the number of TPs, if any [33], on the PO. The position of the SCPs is given by the condition that one of the corresponding manifolds is "vertical." In our case, we have used local coordinates, one parallel and another transverse to the guiding $\mathrm{PO}$, to locate these points. The corresponding positions have been indicated in Fig. 1, with blue circles and red crosses, depending on whether they are generated by the rotation of the stable or unstable manifold, respectively.

The smoothing process implicit in the integration on Eq. (3) renders a function with the probability density well localized along the PO. Furthermore, this localization effect is maximized when $\gamma_{t}$ becomes periodic, by constructive interference, over the PO. This happens when the phase fulfills the $\mathrm{BS}$ quantization rule

$$
\gamma=\frac{S\left(E_{n}\right)}{\hbar}-\frac{\pi}{2} \mu=2 \pi n, \quad n=0,1, \ldots,
$$

where $n$ is an integer giving the number of nodes or excitations along the scarring PO in the wave function, and $\gamma, S$, and $\mu$ are defined over one period of the PO; that is, $\gamma=\gamma_{T}$ and 
$S=S_{T}$. Notice that the usual semiclassical quantization rule $S=2 \pi \hbar(n+\mu / 4)$ is easily recovered from this expression. Moreover, condition (5) is satisfied only for some (semiclassically allowed [34]) values of the energy, $E_{n}$.

The tube functions described above can be improved by incorporating into them the dynamical effects produced by the hyperbolic motions in the neighborhood of the scarring or guiding PO [21]. This can be done, in a convenient way, by propagation of the tube functions followed by a suitable Fourier transformation at the BS quantized energy

$$
\psi_{n}^{\mathrm{scar}}(x, y)=\int_{-T_{E}}^{+T_{E}} d t \cos \left(\frac{\pi t}{2 T_{E}}\right) e^{i\left(E_{n}-\hat{H}\right) t / \hbar} \psi_{n}^{\text {tube }}(x, y) .
$$

Although not absolutely necessary, we have included here a cosine window in order to minimize the dispersion in energy [35]. Notice that we have not used in Eq. (6) the long-time quantum dynamics of $\psi_{n}^{\text {tube }}(x, y)$, since this brings undesired (too complicated) dynamical information into our scar function. Instead, we have considered in the construction of the scar function the dynamics of the system up to a shorter time, chosen as the Ehrenfest time, $T_{E}$. This time is (semiclassically) defined as the lapse in which a Gaussian wave packet spreads and reaches the limits of a characteristic area, $A$, in the Poincaré SOS of the system, and it can be related to the Lyapunov exponent $\left(\bar{\lambda}=0.3848 E^{1 / 4}\right.$ in our case) in the following way: $T_{E}=(1 / 2 \bar{\lambda}) \ln (A / \hbar)$.

Finally, let us remark that the probability density for $\psi_{n}^{\text {scar }}$ is not only localized (in phase space) on the fixed point corresponding to the PO, but also in short pieces of the emanating manifolds, as thoroughly discussed, for example, in Ref. [25].

\section{RESULTS}

Let us examine now the performance of the method that has been just described in the case of the classically chaotic quartic potential, by presenting some results obtained for the POs shown in Sec. II.

The first point that we have to address is the consideration of the symmetry of the computed functions. The quantum eigenstates of the quartic oscillator (1) can be classified according to the $C_{4 v}$ symmetry group, which has five irreducible representations: $A_{1}, B_{1}, A_{2}, B_{2}$, and $E$. For practical purposes this determines the conditions, Dirichlet $(\psi=0)$ or Neumann $\left(\partial_{\perp} \psi=0\right)$, at the axis $(x, y=0)$ and the diagonals $(x= \pm y)$. An elegant way to translate this into semiclassical arguments is to consider the desymmetrized PO resulting from "folding" the original one into the fundamental domain. For the case of the quartic potential, this domain consists, in the case of the one-dimensional representations $A, B$, of the $1 / 8$ region enclosed by one semiaxis and the neighbor semidiagonal, and the $1 / 4$ region between the two semiaxes for the two-dimensional one, $E$. Notice that this procedure introduces "artificial" hard wall boundaries in both the axis and the diagonals having two effects. First, it reduces the length, and then the topological (without the contributions arising from the desymmetrization) and mechanical actions in Eq. (5), in an integer factor of $p$, given by the ratio between the periods of the full and desymmetrized POs. Second, it has an additional more complicated effect in the Maslov index, which is different for the Dirichlet and Neumann cases, that has to be carefully taken into account, as fully discussed in Ref. [22]. Fortunately, the final result is particularly simple for the totally symmetric wave functions, that is, those with Neumann conditions both along the axis and the diagonals. In this case the contributions at the boundaries cancel, and the net effect of the desymmetrization in the quantization condition can be simply accounted for with the factor $p$,

$$
\gamma=\frac{S\left(E_{n}\right) / p}{\hbar}-\frac{\pi}{2}(\mu / p)=2 \pi n, \quad n=0,1, \ldots,
$$

where the quantum number $n$ has been redefined as referring to the fundamental domain. Accordingly, and in order to keep the examples in this paper as simple as possible, we restrict our presentation to the fully symmetric $A_{1}$ case. The generalization to the other symmetry classes is slightly more complicated but straightforward.

We now present our results for the "loop" PO; see Fig. 1. In this case, $\mu=12, p=4$, and then the quantization condition for the total action of the PO reads

$$
S\left(E_{n}\right)=2 \pi \hbar(4 n+3), \quad n=0,1, \ldots
$$

Considering that $S(E=1)=25.0018$ and setting $\hbar=1$,

$$
E_{n}=0.1586(4 n+3)^{4 / 3}, \quad n=0,1, \ldots
$$

In the bottom panels of Fig. 2 we present the scar functions localized over this PO for $n=5$ to 7. As can be seen, and despite the complexity of the guiding PO in configuration space, the probability density of the computed function localizes very well along it, which is an indication of the brilliant performance of our numerical method. Another important characteristic of our functions can be deduced from their spectra, that can be conveniently computed by projection over the eigenstates, $|i\rangle$, of the system. These states were obtained by diagonalization of the Hamiltonian operator corresponding to Eq. (1) in a large basis set consisting of 5000 symmetry-adapted harmonic oscillator eigenfunctions. At infinite resolution these spectra are given by $I_{\infty}(E)=$
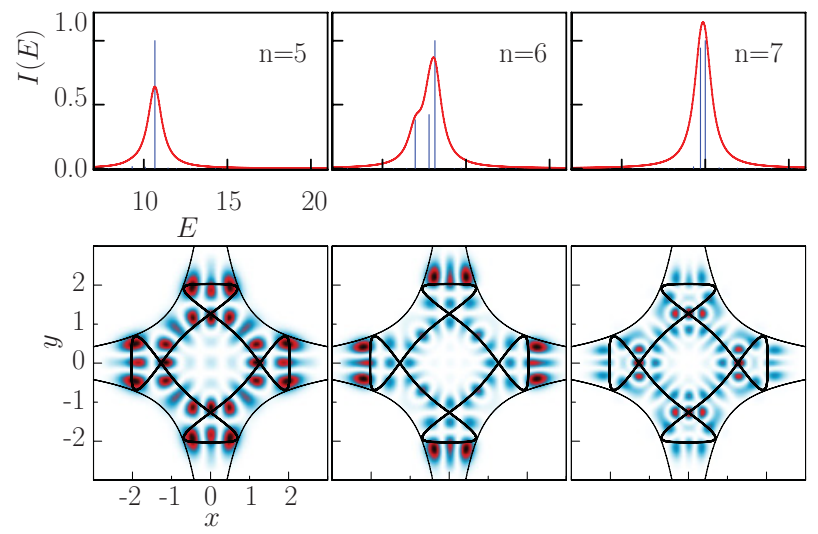

FIG. 2. (Color online) Bottom panel: Probability density for the scar wave function given by Eq. (3) constructed over the "quadrupleloop" periodic orbit (superimposed in black) with quantum numbers $n=5$ to 7 . The plot has been scaled to $E=1$ using the relations in (2). Top panel: Corresponding infinite resolution (sticks) spectra and finite resolution version (continuous line) obtained by convolution with a Lorentzian function of width $\Gamma=0.5$. 


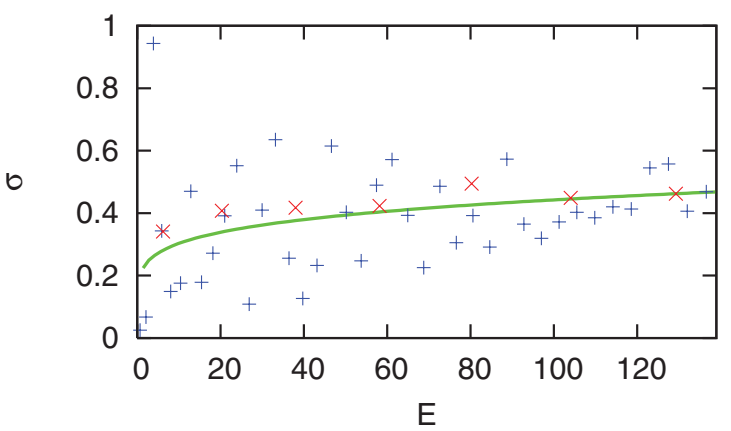

FIG. 3. (Color online) Dispersion of the scar functions along the "quadruple-loop" periodic orbit (blue plus signs). To observe the tendency, the corresponding mobile mean is also plotted (red crosses). The results obtained with the semiclassical approximation (10) are plotted superimposed in green continuous line.

$\sum_{i}\left|\left\langle i \mid \psi_{n}^{\text {scar }}\right\rangle\right|^{2} \delta\left(E-\epsilon_{i}\right)$. The corresponding results are given in the top panel of the figure. As can be seen, our scar functions are not only localized in configuration space but also in energy, since the spectra consist of series of sticks closely packed around the corresponding BS energy values. To emphasize this localization we have also plotted superimposed in the figure the associated finite resolution spectra, $I_{\Gamma}(E)$, obtained by convolution with a Lorentzian of width $\Gamma=5$.

This conclusion is of more general validity, as deduced from the results presented in Fig. 3, where the values for the dispersion, $\sigma_{n}=\left\langle\psi_{n}^{\text {scar }}\left|\left(\hat{H}-E_{n}\right)^{2}\right| \psi_{n}^{\text {scar }}\right\rangle$, of the 40 low-lying scar functions over the "quadruple-loop" PO are displayed (blue plus signs) as a function of the corresponding energies. As can be seen the results are very small and grow very moderately with the energy, this further confirming our previous conclusion on the high localization in energy of the scar function. Moreover, this dispersion fluctuates violently, and then the underlying tendency is more clearly observed in the mobile mean, which is also plotted (red crosses) in the figure. This mean is well approximated semiclassically as [35]

$$
\sigma=\frac{\pi}{2} \frac{\hbar \lambda\left(\zeta+\lambda T_{\mathrm{E}}\right)}{\zeta^{2}+\left(\zeta+\lambda T_{\mathrm{E}}\right)\left(z+\lambda T_{\mathrm{E}}\right)}
$$

where $\lambda$ is the stability index of the PO, $z \approx 1.06078$ and $\zeta=\pi / \sqrt{2}-z \approx 1.16066$.

To conclude this section, we present here our results for the other POs shown in Fig. 1. The corresponding general BS quantization conditions can be written as

$E_{n}=\left[\frac{S\left(E_{n}\right)}{S(E=1)}\right]^{4 / 3}$, with $S\left(E_{n}\right)=2 \pi \hbar\left(p n+\frac{\mu}{4}\right)$,

and the values for $p, \mu$, and $S(E=1)$ are $2,4,12.7134$ for the PO in Fig. 1(a), 1, 6, 17.8311 for the PO in Fig. 1(b), 1, 6, 15.4243 for the PO in Fig. 1(c), and 2, 8, 17.0268 for the PO in Fig. 1(d), respectively.

Again, it should be remarked that these POs have been chosen since they represent complicated figures in configuration space, which in principle do not help in the application of any scar function computation method.

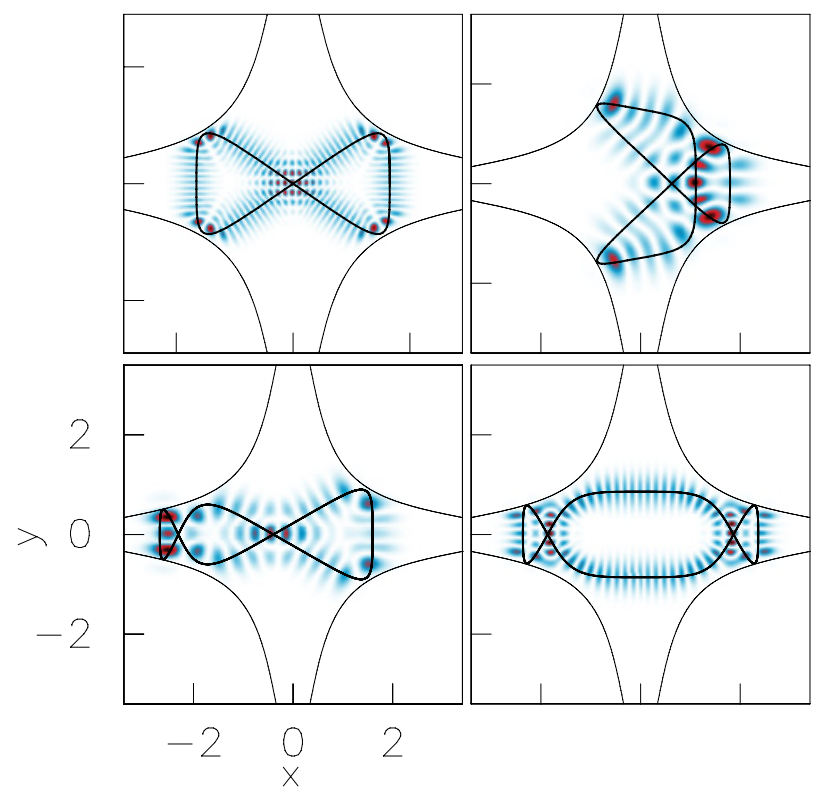

FIG. 4. (Color online) Same as Fig. 2 corresponding to $n=20$ for the other periodic orbits presented in Figs. 1(b)-1(e).

Our results for the corresponding scar functions with $n=$ 20 and $\hbar=1$ are shown in Fig. 4. Notice that although they all correspond to the same quantum number (in the fundamental domain) they do not exhibit the same number of total nodes. This is due to the fact that the different desymmetrized POs which are quantized have different lengths due to symmetry reasons. Actually, in the first (top left panel) and last cases (bottom right panel) one fourth of the original PO is to be taken in the calculation, while in the other two cases (top right and bottom left panels) one half of it is needed. Also notice that these wave functions do not exhibit the correct $C_{4 v}$ symmetry of the system, something that can be easily solved by obtaining the symmetry adapted linear combinations of the corresponding scar functions obtained from the POs that result from repeated application of the $C_{4}$ symmetry operator. Finally, it should be commented that all the results presented in Fig. 4 convincingly show that our method has no problem in computing wave functions with the probability density highly localized on the POs, also having the correct number of nodes along them.

\section{SUMMARY}

In this paper, we have checked the performance of a simple and straightforward method to efficiently compute in a systematic way scar functions along unstable POs. The procedure consists of averaging the dynamics of a wave packet forced to stay in the immediate neighborhood of a guiding $\mathrm{PO}$, and imposing the correct quantization condition for the action. An application to some a priori complicated POs of a classically chaotic two-dimensional quartic potential, which constitutes an ideal benchmark for studies of quantum chaos, is presented as an illustration. In all cases, the computed wave functions appear highly localized along the selected POs having the desired number of nodes. This fact is an indication of the excellent performance of the proposed method. Finally, our functions are shown to be also well localized in the 
energy domain. This is an important point, which indicates that they are suitable candidates to be used as a good dynamical basis set for the efficient computation of eigenstates of this kind of system. We have actually verified this possibility, and the corresponding results will be published in the near future [36].

\section{ACKNOWLEDGMENTS}

Support from MICINN-Spain under Contracts No. MTM2009-14621 and No. i-MATH CSD2006-32, and from CEAL Banco de Santander-UAM is gratefully acknowledged. F.R. thanks the UPM for support through a doctoral fellowship.
[1] M. C. Gutzwiller, Chaos in Classical and Quantum Mechanics (Springer Verlag, New York, 1990).

[2] A. Einstein, Verh. Dtsch. Phys. Ges. 19, 82 (1917).

[3] A. D. Stone, Phys. Today 58(8), 37 (2005).

[4] M. C. Gutzwiller, J. Math. Phys. 11, 1791 (1970).

[5] E. J. Heller, Phys. Rev. Lett. 53, 1515 (1984).

[6] E. J. Heller, in Chaos and Quantum Physics, edited by M. J. Giannoni, A. Voros, and J. Zinn-Justin (Elsevier, Amsterdam, 1991).

[7] L. Kaplan and E. J. Heller, Ann. Phys. (NY) 264, 171 (1998).

[8] E. B. Bogomolny, Physica D 31, 169 (1988).

[9] M. V. Berry, Proc. R. Soc. London A 423, 219 (1989).

[10] S. Tomsovic and E. J. Heller, Phys. Rev. Lett. 70, 1405 (1993); S. Tomsovic and J. H. Lefebvre, ibid. 79, 3629 (1997).

[11] D. A. Wisniacki, E. Vergini, R. M. Benito, and F. Borondo, Phys. Rev. E 70, 035202(R) (2004); Phys. Rev. Lett. 94, 054101 (2005); 97, 094101 (2006).

[12] J. P. Keating and S. D. Prado, Proc. R. Soc. London A 457, 1855 (2001).

[13] D. Wisniacki and G. G. Carlo, Phys. Rev. E 77, 045201(R) (2008); M. Novaes, J. M. Pedrosa, D. Wisniacki, G. G. Carlo, and J. P. Keating, ibid. 80, 035202(R) (2009).

[14] H. J. Stöckman, Quantum Chaos. An Introduction (Cambridge University Press, Cambridge, UK, 2000).

[15] S. Sridhar, Phys. Rev. Lett. 67, 785 (1991); J. Stein and H. Stöckmann, ibid. 68, 2867 (1992).

[16] P. B. Wilkinson et al., Nature (London) 380, 608 (1996); R. Akis, D. K. Ferry, and J. P. Bird, Phys. Rev. Lett. 79, 123 (1997).

[17] J. U. Nöckel and A. D. Stone, Nature (London) 385, 45 (1997); C. Gmachl, F. Capasso, E. E. Narimanov, J. U. Nöckel, A. D. Stone, J. Faist, D. L. Sivco, and A. Y. Cho, Science 280, 1556 (1998); S.-B. Lee, J.-H. Lee, J.-S. Chang, H.-J. Moon, S. W. Kim, and K. An, Phys. Rev. Lett. 88, 033903 (2002); T. Harayama, T. Fukushima, P. Davis, P. O. Vaccaro, T. Miyasaka, T. Nishimura, and T. Aida, Phys. Rev. E 67, 015207(R) (2003); Q. H. Song, L. Ge, A. D. Stone, H. Cao, J. Wiersig, J.-B. Shim, J. Unterhinninghofen, W. Fang, and G. S. Solomon, Phys. Rev. Lett. 105, 103902 (2010); J. Wiersig, A. Eberspächer, J.-B. Shim, J.-W. Ryu, S. Shinohara, M. Hentschel, and H. Schomerus, Phys. Rev. A 84, 023845 (2011).
[18] V. Doya, O. Legrand, F. Mortessagne, and C. Miniatura, Phys. Rev. Lett. 88, 014102 (2001); C. Michel, V. Doya, O. Legrand, and F. Mortessagne, ibid. 99, 224101 (2007).

[19] L. Huang, Y.-C. Lai, D. K. Ferry, S. M. Goodnick, and R. Akis, Phys. Rev. Lett. 103, 054101 (2009).

[20] G. G. de Polavieja, F. Borondo, and R. M. Benito, Phys. Rev. Lett. 73, 1613 (1994).

[21] E. G. Vergini, J. Phys. A 33, 4709 (2000); E. G. Vergini and G. G. Carlo, ibid. 33, 4717 (2000).

[22] E. G. Vergini and G. G. Carlo, J. Phys. A 34, 4525 (2001); G. G. Carlo, E. G. Vergini, and P. Lustemberg, ibid. 35, 7965 (2001).

[23] A. Vagov, H. Schomerus, and V. V. Zalipaev, Phys. Rev. E 80, 056202 (2009).

[24] E. J. Heller, P. W. O’Connor, and J. Gehlen, Phys. Scr. 40, 354 (1989).

[25] E. L. Sibert III, E. Vergini, R. M. Benito, and F. Borondo, New J. Phys. 10, 053016 (2008).

[26] E. G. Vergini, E. L. Sibert III, F. Revuelta, F. Borondo, and R. M. Benito, Europhys. Lett. 89, 40013 (2009).

[27] R. L. Waterland, J.-M. Yuan, C. C. Martens, R. E. Gillilan, and W. P. Reinhardt, Phys. Rev. Lett. 61, 2733 (1988); C. C. Martens et al., J. Chem. Phys. 90, 2328 (1989); B. Eckhardt, G. Hose, and E. Pollak, Phys. Rev. A 39, 3776 (1989); O. Bohigas, S. Tomsovic, and D. Ullmo, Phys. Rep. 223, 44 (1993).

[28] P. Dahlqvist and G. Russberg, Phys. Rev. Lett. 65, 2837 (1990).

[29] E. J. Heller, J. Chem. Phys. 65, 4979 (1976).

[30] R. G. Littlejohn, Phys. Rep. 138, 195 (1986).

[31] V. Maslov and M. Fedoriuk, Semiclassical Approximation in Quantum Mechanics (Reidel, Dordrecht, 1981).

[32] S. C. Creagh, J. M. Robbins, and R. G. Littlejohn, Phys. Rev. A 42, 1907 (1990); J. M. Robbins, Nonlinearity 4, 343 (1991).

[33] Notice that all considered POs present no TPs.

[34] M. Brack and R. M. Bhaduri, Semiclassical Physics (AddisonWesley, Reading, 1997).

[35] E. Vergini, D. M. Schneider, and A. M. F. Rivas, J. Phys. A 41, 405102 (2008).

[36] F. Revuelta, R. M. Benito, F. Borondo, and E. Vergini (unpublished). 